\title{
ANALYTIC HIERARCHY PROCESS AND AGENT-BASED SIMULATION FOR TRAFFIC MODELING
}

\author{
Richard Cimler \\ Faculty of Informatics and Management \\ University of Hradec Králové \\ Hradec Králové, Czech Republic \\ E-mail: richard.cimler@uhk.cz
}

\begin{abstract}
We present an agent-based model of traffic at the roundabout. The model was implemented in agent based programming language NetLogo. Agents in the model correspond to cars. Each car has got its parameters and behavior. The behavior is expressed using multi-criteria decision making schema and analytic hierarchy process. Application of analytical hierarchy process in multi agent system is described. Driver's decision depends on his own character attributes as well as on driver's current situation. It is possible to experiment with different traffic situations and to observe the impact of each setting on the fluency of the traffic.
\end{abstract}

Keywords: traffic model, NetLogo, multi-criteria decision making

\section{Introduction}

Our research is motivated by a real traffic problem in our city (Hradec Kralove, Czech Republic). The road system of the city consists of two main homocentric circles and radial roads. The object of our interest is situated in the outer circular road (Fig. 1). This heavily used intersection is planned to be replaced with a system of two roundabouts (Fig. 2) to make traffic more fluent and eliminate traffic congestions.

Current intersection is managed by traffic lights. The average number of vehicles per day is nearly 30000 on the west side and about 20000 on the east side of the intersection. Moreover there is a large hospital situated next to the intersection, with ambulances moving out frequently and having to cross the intersection with the highest priority. There are differences in number of vehicles coming from each of four directions. West-east (and east-west) road is the most frequent because of the transit traffic. Westnorth and south-north directions are typically used by local drivers going to the city center. West-north traffic is higher than east-north because of the radial road at the adjacent intersections.

To compare the effectiveness of both solutions (intersection versus roundabouts), we designed an agentbased simulation model. The model is implemented in NetLogo (Wilensky, 1999) which can be used for simulating different situations such as (Cimler, 2012). The objective of our agent-based model is to enable experiments to test two hypotheses:

- the roundabout makes the traffic more fluent,

- the system of two roundabouts enables faster through passage of ambulances.

The model is based on the data gathered from the maps, observations and measurement of the traffic lights switching intervals. Agents correspond to drivers (and their cars). Each driver has got its own 
parameters and behavior. The behavior is expressed using multi-criteria decision making schema and analytic-hierarchy process. Several types of drivers are defined and the ratio of each driver's type can be changed. It is possible to experiment with fractions s of cars, lorries, buses or ambulances together with a mixture of more or less patient, rude, experienced or cautious drivers.

The traffic density is the main parameter of the model. It can be managed using control elements in the model interface. Each setting of model parameters corresponds to a particular traffic situation. Simulation experiments help to observe the impact of each setting (crossing, roundabout) on the total fluency of the traffic in the location.

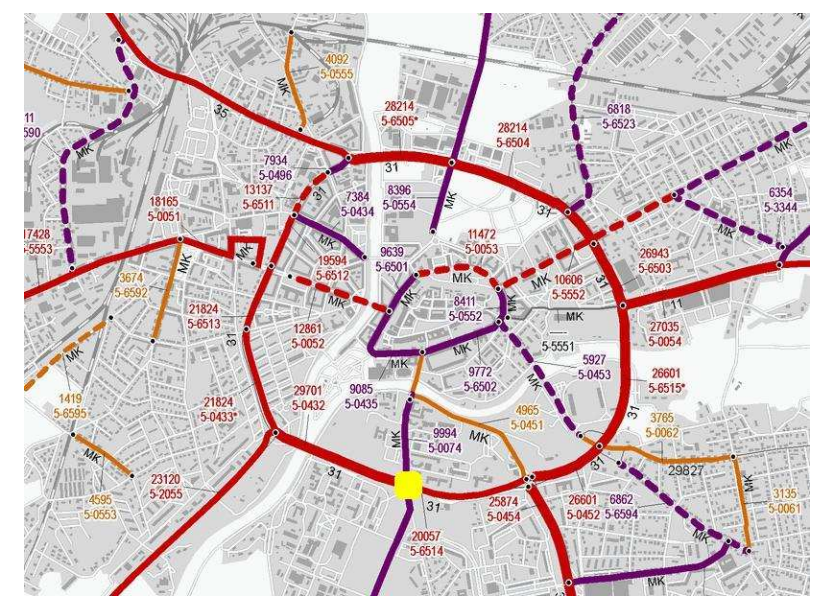

Fig. 1 Road transport in Hradec Kralove

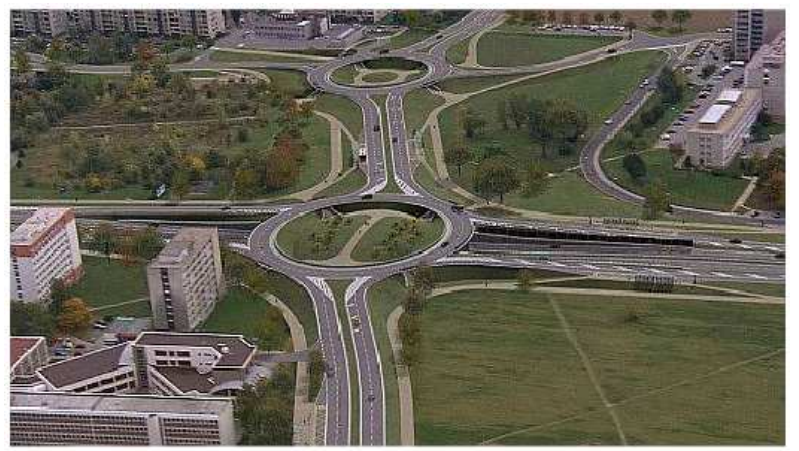

Fig. 2. Visualization of the planned system of two roundabouts

\section{Model description}

Each agent-car has its own characteristic. Basic characteristics of the car are maximum speed, acceleration, view distance, current speed, and destination. Cars are of three types: normal vehicles, trucks and ambulances. The difference between normal vehicles and trucks is maximum speed and acceleration parameters. Ambulances are cars with the highest priority- other cars have to stop if the ambulance is passing around. 
There are 3 types of road: normal way, entrance and roundabout. Entrance is part of the way near to the roundabout. Driver has to prepare there for entering the roundabout. Drivers have different driving rules due to the type of the road.

Drivers are following marks in the simulation and trying to reach their destination. Navigation in the simulation is provided by marks which are of six types: (1) start point of entrance, (2) start point of roundabout, (3) end point of entrance, (4) exit, (5) destination and (6) start of tunnel. Part of the simulation is on the fig.3.

- Start point of entrance is mark telling driver to prepare for entering the roundabout - decrease speed.

- Start of roundabout is most important mark. All decisions are made by drivers who reach this mark. Decision process will be described in the following part of the paper.

- End point of entrance is place on the roundabout which drivers head to as they entering roundabout. At the moment driver reach this mark he change type of his movement due to algorithms created for moving on the roundabout.

- Exit is the mark near the exit of the roundabout and contains information about destinations in the direction of this exit. If there is information about drivers' destination, driver leaves the roundabout on this exit.

- Destination is mark showing driver that he reaches his destination as he reaches this mark. Statistics about this driver are saved and then driver deleted.

- If the driver reaches start of tunnel mark the car starts to be invisible for other drivers as well as for user. Car became visible as it reaches for the other mark start of the tunnel.

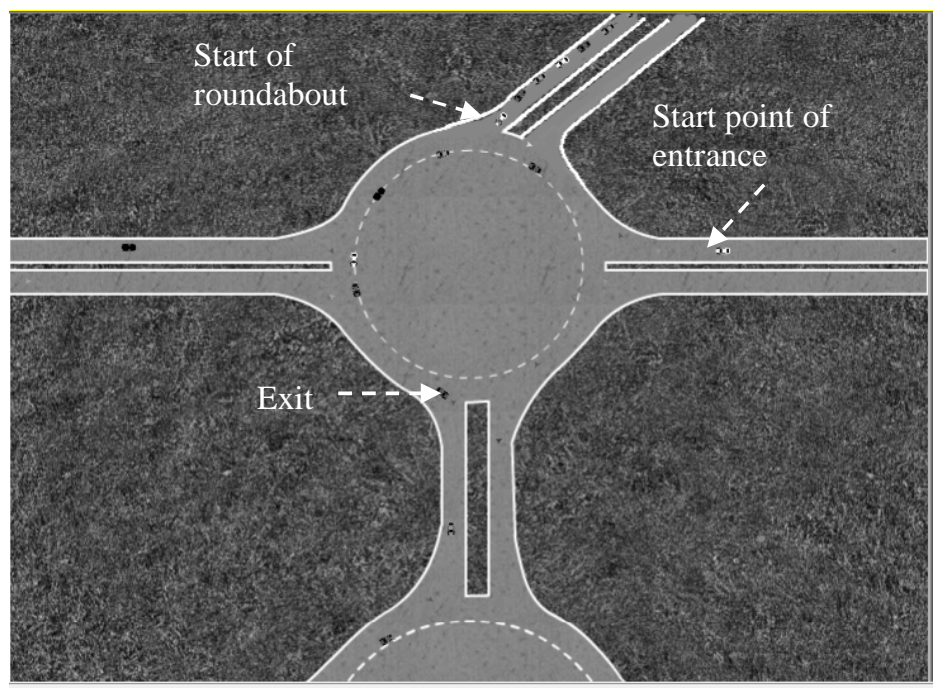

Fig. 3. NetLogo model of the roundabout

\section{Driver's decision making}

Many factors affect driver's decision. In the basic model of the traffic only proximity to the other car on the roundabout arbitrates driver's actions. All other factors which in a real life influence driver's decision were neglected. Driver, as he reaches start of roundabout mark, has to decide if he wants to enter the roundabout or wait. There was a simple rule: if the distance to the other car (which is already on the roundabout and can cross driver's trajectory) is smaller than a safe distance (set as a parameter in the start of the simulation) then driver waits and do not continue in his driving. This rule is too much simple and that is why agent based model was extended by multi-criteria decision making. 
Each driver has its own criteria matrix depending on his character. In the moment when driver is deciding about his next action, situation vector (describing driver's current situation) is created. Using the decision making method - AHP, described below, driver chooses the action which should be best for him. Our model is presented on the following example. Number of criteria can increase as well as situation vector can be more comprehensive.

\subsection{AHP}

Principles of AHP were formulated by Saaty in late 1970s (Saaty, 1980). This method helps to create structured models of multi-criteria decision problems. Problem is divided into the sub problems and each sub problem is analyzed separately. There can be many criteria connected to the decision making process. To create ranking of these criteria pair-wise comparison is used. Every two criteria are compared and relative importance of each is set. Result values are in the comparison matrix A.

In AHP approach an $\mathrm{n} x \mathrm{n}$ pair-wise comparison matrix A (criteria matrix) with positive elements $\mathrm{a}_{\mathrm{ij}}$ is considered (Mls, 2013)

$$
A=\left[\begin{array}{cccc}
1 & a_{12} & \cdots & a_{1 n} \\
a_{21} & 1 & \cdots & a_{2 n} \\
\vdots & \vdots & \ddots & \vdots \\
a_{n 1} & a_{n 2} & \cdots & 1
\end{array}\right] .
$$

This matrix is reciprocal, so $\mathrm{a}_{\mathrm{ij}}=1 / \mathrm{a}_{\mathrm{ji}}$ for each $1 \leq \mathrm{i}, \mathrm{j} \leq \mathrm{n}$.

Normalised principal eigenvector of the matrix $\mathrm{A}$ is computed to get priorities of respective alternatives.

We say that $A$ is consistent, if $a_{i j} \cdot a_{j k}=a_{i k}$ for each $1 \leq i, j, k \leq n$.. If $a_{i j} \cdot a_{j k} \neq a_{i k}$ for some $i, j, k$, than $A$ is said to be inconsistent. In AHP, it is assumed that $1 / 9 \leq \mathrm{a}_{\mathrm{ij}} \leq 9$., for all $1 \leq \mathrm{i}, \mathrm{j} \leq \mathrm{n}$. The inconsistency of $A$ is measured by the consistency index $\mathrm{CI}_{\mathrm{n}}$ defined as

$$
C I_{n}=\frac{\lambda_{\max }-n}{n-1},
$$

where $\lambda_{\max }$ is the principle eigenvalue of $\mathrm{A}$. If $\mathrm{A}$ is an $\mathrm{n} \mathrm{x} \mathrm{n}$ positive reciprocal matrix, then $\mathrm{A}$ is consistent if $\mathrm{CI}_{\mathrm{n}}=0$. Inconsistency index is discussed also in (Ramík and Korviny, 2009).

By computing an eigenvector $\mathrm{X}$ of the matrix A relative rankings of variables can be obtained.

$$
\mathrm{AX}=\lambda \mathrm{X}
$$

Final ranking of particular alternatives is computed as matrix multiplication of eigenvector of $X$ and matrix describing possible alternatives.

\section{Example}

Simulation created in NetLogo allows us to obtain a lot of information about driver's status and its surroundings. Some of the information which is possible to obtain is number and speed of other cars, proximity to crossing, proximity to other car etc. Based on this information driver can decide about his next action. 


\subsection{Criteria matrix}

Criteria matrix (pair-wise comparison matrix A) is based on the attributes of drivers. Criteria matrix consists of the 3 criteria - number of the criteria can increase while the model will be extended. Driver's attributes are divided into two parts. Driver can be patient or impatient and experienced or inexperienced.

Criteria matrix consists of:

- Proximity - In this criterion, reaction on the car which is already on the roundabout and can cross driver's trajectory is described. Cautious drivers tend to stop if the other car is approaching. In contrast to rude drivers who will in the same situation continue driving or increase their speed. Proximity is very important for inexperienced drivers.

- Rush - This criterion shows if the driver is in a hurry. This criterion is important for impatient drivers.

- Environment - Expresses caution of the drivers if weather conditions and conditions of the road are not good. For inexperienced drivers is this criterion important.

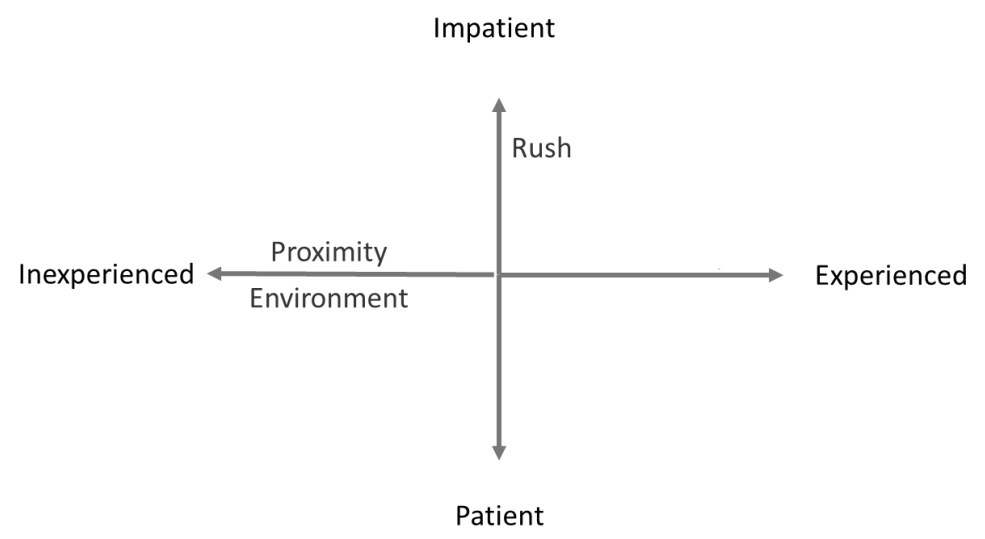

Fig. 4. Driver`s attributes

\subsection{Situation vectors}

Situation vector is written as [stop; go normal; go fast]. It represents current situation of the driver. There are three types of situation vectors in our example: environment vector, rush vector and proximity vector. Vector doesn't describe driver's character; it only describes his current situation and is recommendation how to behave.

\subsection{Environment}

At the begging of the simulation random environment status is generated. In our example environment status is static for the entire simulation. The future enhancements will include the possibility of modifying the environment status, which is going to enable other scenarios such as alternation of day and night or changing of the weather. 


\subsection{Proximity}

Based on the proximity of the other car on the roundabout this vector is created. If the other car is far, driver can continue driving and enter the roundabout. If other car is near, driver should increase his speed and enter the roundabout fast or can also stop and wait.

\subsection{Final decisions}

While driver approaching to the roundabout he has to choose from three possible actions. Decision depends on driver's character (criteria - comparison matrix) and on the current situation on the roundabout. In previous section driver's criteria matrix and situation vector has been introduced. Possible actions are to continuing driving normally, stopping or increasing speed and enter quickly the roundabout.

Driver doesn't make mistakes in his decision. Result of the computation is final, driver will do recommended action.

\subsection{Computation}

Driver is entering to the simulated area and his criteria (comparison) matrix $\mathrm{A}(\mathrm{n} \times \mathrm{n})$ and eigen-vector $\mathrm{x}$, where the $x_{-} i$ respresents the input of $i$-th row, of this matrix is created. While is driver deciding about his next action, situation column vectors $v(i)$, where the $i$ is a number of type of the situation, $i=1$ to $n$, are created. The column inputs of result matrix $B$ are created as: for every $\mathrm{j}$ from 1 to $\mathrm{n}$ and for every $\mathrm{i}$ from 1 to $n: B \_j=v(i)^{*} x \_i$ hold true. Column vector $s(j): j=1 . . n$, for final decision $f$ is $\sum$ for every $i$ from 1 to $n$ of $b_{-}\{i j\}$.

\section{Experiment}

One of many possible settings of the model will be shown in this experiment. We have decided to have two types of drivers. First type is patient and inexperienced drivers and second type is impatient and experienced drivers. For this example, criteria matrices have been chosen as following. This an example of way how can be model used. Creation of the matrices describing more realistic values for specific situation will be work for expert team and will be used in our future research.

Table 1. Patient and inexperienced

\begin{tabular}{|c|c|c|c|}
\hline \multirow{3}{*}{$\begin{array}{c}\text { Proximity } \\
\text { Rush }\end{array}$} & Proximity & Rush & Environment \\
\hline & 1 & 8 & 2 \\
\hline & $1 / 8$ & 1 & $1 / 7$ \\
\hline Environment & $1 / 2$ & 7 & 1 \\
\hline
\end{tabular}

Table 2. Impatient and experienced

\begin{tabular}{|c|c|c|c|}
\hline \multirow{3}{*}{$\begin{array}{l}\text { Proximity } \\
\text { Rush }\end{array}$} & Proximity & Rush & Environment \\
\hline & 1 & 2 & 2 \\
\hline & $1 / 2$ & 1 & 2 \\
\hline Environment & $1 / 2$ & $1 / 2$ & 1 \\
\hline
\end{tabular}


Situation vector is created as follows. Vector is written as [stop] [normal] [fast] (sum is equal to 1) in the lines of following matrices. Appropriateness of each action (stop, go normal, go fast) depends on the current situation. For example in table 3: If other car's proximity is moderate it is appropriate to stop and do not enter the roundabout or increase speed and enter it fast before other car can cross our trajectory. It is not appropriate to enter the roundabout normal speed because there is a bigger chance of crash.

Table 3. Proximity vectors

\begin{tabular}{c|c|c|c|}
\multicolumn{1}{c}{} & \multicolumn{1}{c}{ Stop } & \multicolumn{1}{c}{ Normal } & Fast \\
\cline { 2 - 4 } Far & 0.05 & 0.8 & 0.15 \\
\cline { 2 - 4 } Moderate & 0.7 & 0 & 0.3 \\
\cline { 2 - 4 } Small & 1 & 0 & 0 \\
\cline { 2 - 4 } & &
\end{tabular}

Table 4. Rush vectors

\begin{tabular}{|c|c|c|c|}
\hline \multirow{4}{*}{$\begin{array}{c}\text { Far } \\
\text { Moderate } \\
\text { Small }\end{array}$} & Stop & Normal & Fast \\
\hline & 0 & 0.3 & 0.7 \\
\hline & 0.2 & 0.4 & 0.4 \\
\hline & 0.3 & 0.7 & 0 \\
\hline
\end{tabular}

Table 5. Environment vectors

\begin{tabular}{c|c|c|c|}
\multicolumn{1}{c}{} & \multicolumn{1}{c}{ Stop } & Normal & Fast \\
\cline { 2 - 4 } Far & 0 & 0.5 & 0.5 \\
\cline { 2 - 4 } Moderate & 0.3 & 0.5 & 0.2 \\
\cline { 2 - 4 } Small & 0.5 & 0.4 & 0.1 \\
\cline { 2 - 4 } & &
\end{tabular}

\subsection{Results}

Each setup has been run for ten times and results are average values. Impact of the environment status has been tested on patient-inexperienced drivers and impatient-experienced drivers. Environment status has been set to bad, moderate and good. Rush status has been set to moderate for all of the drivers.

All drivers do not risk getting fast to the roundabout if the environment is not good.

If the environment is good, impatient and experienced drivers made decision go fast in $7 \%$ of decision making situations Patient and inexperienced drivers prefer to stop even if the environment is good. Results of driver's decisions can be seen in the table 6 .

Table 6. Drivers decisions in good environment

Patient and inexperienced

Impatient and experienced

\begin{tabular}{|c|c|c|}
\multicolumn{1}{c}{ Fast } & Normal & \multicolumn{1}{c}{ Stop } \\
\hline $0 \%$ & $6 \%$ & $94 \%$ \\
\hline $7 \%$ & $28 \%$ & $65 \%$ \\
\hline
\end{tabular}




\section{Conclusion}

Agent based model of the roundabout containing agents using multi-criteria decision has been introduced. Model is prepared for the discussion about setting new different types of drivers and their criteria matrices. Lot of information can be gained from the surroundings of the agent. This information can be used in the decision making process. Some of the information has been shown in the example. In our future research new features will be added such as a changing of day and night or changing of the weather. These features affect driver's decision about their next action. Condition of the vehicle can be also considered in the decision making process. Agent based simulation can also provide useful information about problematic locations and creation of the traffic jams on the crossings and roundabouts.

\section{REFERENCES}

Cimler, R., Kautzká, E., Olševičová, K., Gavalec, M. (2012) Agent-based Model for Comparison of Aircraft Boarding Methods. Mathematical Methods in Economy.

Mls, K., Otčenášková, T. (2013) Analysis of Complex Decisional Situations in Companies with the Support of AHP Extension of Vroom-Yetton Contingency Model. IFAC MIM '2013 International Conference, Sain Petersburg, Russia

Ramík, J. and Korviny, P. (2009). Inconsistency of pair-wise comparison matrix with fuzzy elements based on geometric mean. Fuzzy Sets and Systems, 161, pp.1604-1613.

Saaty, T.L. (1980). The analytic hierarchy process. McGraw-Hill, New York.

Wilensky, U. (1999). NetLogo. http://ccl.northwestern.edu/netlogo/. Center for Connected Learning and Computer-Based Modeling, Northwestern University, Evanston, IL. 